\title{
Mathematical Model On Power Needed To Sustain Flight.
}

\author{
Efor T. E (Mrs) and Ajibo D. I. \\ Department of Industrial Mathematics and Applied Statistics Ebonyi state University, Abakaliki.
}

\begin{abstract}
The purpose of this paper is to use mathematical model to determine the minimum power needed to sustain flight. We considered, parasitic power, minimum velocity and lift power so as to get the minimum power needed to sustain flight and our general model is of the form, $P_{T}=P_{L}+P_{p}$. We efficiently utilized the Newton's laws of motion, Bernoulli's principle, and the Lift coefficient approach method for determining lift, in achieving this model.
\end{abstract}

Key words: Lift coefficient, parasitic power, Minimum velocity, Lift power, Minimum power, and Newton's laws.

\section{Introduction}

A model can be simply defined as a representation of a real system or process. A conceptual model is a hypothesis for how a system or process operates. This hypothesis can be expressed quantitatively as a mathematical model, for instance, $P_{T}=P_{p}+P_{L}$ ). They are abstractions that represent processes as equations, physical properties as constants or coefficients in the equation and measure of state or potential in the system as variable.

In the world today, the rate at which lives are being lost as a result of plane crash is worrisome. The paper is to mathematically model the system and find the minimum power needed for the plane to move without any problem and in doing that, we will be utilizing the lift coefficient approach in getting the lifting force which will be manipulated to get the lift power.

1.1 The lift coefficient approach: The lift coefficient approach is given by

$L=1 / 2 v^{2} \rho a C_{L}$

Where: $L=$ Lift force

$$
\begin{gathered}
v=\text { Air speed } \\
\rho=\text { Air density } \\
a=\text { Platform area } \\
C_{L}=\text { Lift coefficient }
\end{gathered}
$$

From the above, we will get the parasitic power $\left(P_{p}\right)$ by multiplying the parasitic force $\left(F_{p}\right)$ with velocity, and the lift power $\left(P_{L}\right)$ will be gotten in a similar manner using the Newton's law of motion as our foundation. Giving us our general model which is the total power required to sustain flight $\left(P_{T}\right)$ to be the summation of the parasitic power $\left(P_{p}\right)$ which is the result of the counter-balancing of thrust and drag with the Lift power $\left(P_{L}\right)$ which is the result of the counter-balancing of the forces of weight and Lift. The general model is stated as follows: $P_{T}=P_{L}+P_{p}$

\subsection{Weight, Thrust and Lift}

Weight: This simply puts the gravitational force of attraction on any object. This is the force the earth pulls on every object to itself in response to the universal law of gravitation: any two objects because of their masses attract each other with a force that is directly proportional to the product of their masses and inversely proportional to the square of the distance between them. Represented mathematically as:

$$
\begin{aligned}
f & \propto m_{1} m_{2}, \\
f & \propto \frac{1}{d^{2}} m_{2} \\
=f & \propto \frac{m_{1} m_{2}}{d^{2}} \\
\Rightarrow f & =\frac{k m_{1} m_{2}}{d^{2}}
\end{aligned}
$$

In the context of what we are studying, weight refers to the entirety of this pull on the flying object. In other words the weight of a plane comprises of all the parts of the plane.

Thrust: This is one of the four flight forces and is concerned with the forward movement of the flying object. In birds, this force is mainly produced by the wings, as they flap them in such a manner that air is been thrown 
backwards to produce a forward movement of the object (thrust) while some air is been thrown down for the purpose of our next point. But then, in every artificial flying object this force is produced by the engine either directly as in most cases or indirectly as in flapping plane (ornithopter).

Lift: In a simple statement, this is the force that counteracts weight. This is also known as upward thrust. One of the (and the main) way(s) flying objects achieve this is by throwing air downwards.

\section{Methods of Arriving at our Target model}

In this section we will be looking at the different principles, theorems and approach that were efficiently utilized in other to arrive at our target model, they are as follows:

2.1 Newton's Laws: Newton's laws of motion are applicable in flight via the following point:

Deflection: one way to understand the generation of lift in flight is to observe that air is deflected as it passes the airfoil. Since the foil must exert a force on the air to change its direction, the air must as well, exert a force of equal magnitude but in an opposite direction of the foil. In the case of airplane wing, the wings exert a downward force on the air while the air exerts an upward force on the wing. This explanation relies on the second and third Newton's laws of motion.

The net force on an object is equal to its rate of momentum change; and, to every action there is an equal and opposite reaction. Mathematically it can be represented as shown below:

Newton's second law:

$\mathrm{P}=$ momentum

$$
f=\frac{d p}{d t}
$$

Newton's third law (Action-reaction law):

$$
\begin{gathered}
\Rightarrow p=m v \\
\frac{d p}{d t}=\frac{d(m v)}{d t} \\
a=\frac{d v}{d t} \\
=m \cdot \frac{d v}{d t}=m a \\
\Rightarrow f=m a
\end{gathered}
$$

$$
f_{1,2}=-f_{2,1}
$$

Observe that objects 1 and 2 are considered to be in the same system, and then the net force of the system due to interaction is zero.

Alternatively:

$$
\begin{gathered}
\Rightarrow f_{1,2}+f_{2,1}=0 \\
\sum f=0
\end{gathered}
$$

Integrate through with respect to time (t).

$$
\begin{aligned}
f_{1,2} & =\frac{d p_{1,2}}{d t} \\
-f_{2,1} & =-\frac{d p_{2,1}}{d t} \\
f_{1,2} & =-f_{2,1} \\
\Rightarrow \frac{d p_{1,2}}{d t} & =-\frac{d p_{2,1}}{d t}
\end{aligned}
$$

$$
\begin{gathered}
\Delta p_{1,2}=-\Delta p_{2,1} \\
\sum \Delta=\Delta p_{1,2}+\Delta p_{2,1}=0
\end{gathered}
$$

That is to say, that air "turns" as it passes the airfoil and follows a path that is curved. When airfoil changes direction a reacting force is generated opposite to the directional change.

2.2 Bernoulli's Principle: States that the sum of the pressure at any part plus the kinetic energy per unit volume plus the potential energy per unit volume, there is always a constant. The principle is mathematically derived as follows:

Recall: mass $/$ volume $=$ Density

$$
\begin{gathered}
T_{E}=P_{E}+K_{E} \\
\frac{T_{E}}{\text { volume }}=\frac{P_{E}}{\text { volume }}+\frac{K_{E}}{\text { volume }} \\
\Rightarrow p_{1}-p_{2}=\frac{m g\left(h_{2}-h_{1}\right)}{\text { volume }}+\frac{1 / 2 m\left(v_{2}^{2}-v_{1}^{2}\right)}{\text { volume }}
\end{gathered}
$$


Expand and collect like terms, we have:

$$
=\rho g\left(h_{2}-h_{1}\right)+\frac{1}{2} \rho\left(v_{2}^{2}-v_{1}^{2}\right)
$$

$$
\begin{gathered}
p_{1}+1 / 2 \rho v_{1}^{2}+\rho g h_{1}=p_{2}+1 / 2 \rho v_{2}^{2}+\rho g h_{2} \\
\Rightarrow p+1 / 2 \rho v^{2}+\rho g h=\text { constant } \ldots \ldots \ldots \ldots \ldots \ldots \\
=p+1 / 2 \rho v^{2}+\rho g h=0 \\
p=-\left(1 / 2 \rho v^{2}+\rho g h\right)
\end{gathered}
$$

Multiplying through by -1

$$
\Rightarrow-p=1 / 2 \rho v^{2}+\rho g h
$$

$\boldsymbol{\rho} g h=P_{S}$

$$
\text { Let }-\mathrm{P}=P_{T}
$$

Where $\quad T_{E}$ is total energy, $P_{E}$ and $K_{E}$ are potential and kinetic energy respectively, $P_{T}$ and $P_{S}$ are total and static pressure respectively.

This gives us the direct relationship between pressure and velocity. However Bernoulli's principle shows that at points in a moving fluid where the potential energy ( $\boldsymbol{\rho g h})$ change is very small or zero, as in flow through a horizontal domain (pipe), the pressure is low where the velocity is high conversely, the pressure is high where the velocity is low.

From equation * above we have:

Where $\mathrm{h}=0$

$$
p+1 / 2 \rho v^{2}+\rho g h=0
$$

$$
P=-1 / 2 \rho v^{2}
$$

From the above we noticed that an increase in the velocity (v) of the system causes a decrease in pressure and vice versa.

The mathematical explanation above is highly dependent on the following physical reasoning:

* conservation of momentum: This is a direct consequence of the Newton's laws of motion, especially Newton's second law which relates the net force on an element of air to its rate of momentum change.

*conservation of mass: It encompasses the common assumption that an airfoil's surface is impermeable for air particles flowing around.

* conservation of energy: It states that energy is neither created nor destroyed in a closed system.

What this tells us is: in the equation ** above, let $\mathrm{q} \equiv 1 / 2 \boldsymbol{\rho} v^{2}$. By Bernoulli, $\mathrm{q}$ is smaller above the airfoil, but this must be balanced, so by conservation of momentum, a force must be called upon, and this is the pushing force from below. So that from the conservation of mass and energy, the wing/airfoil is pushed upwards causing the lift. ( $\mathrm{q}$ is called the dynamic pressure).

\subsection{Determining Lift On An Airfoil.}

Here we shall be considering mathematically the methods of determining lift on an airfoil. In doing this we will be basically looking at three different approaches. It shall be seen as the resultant work done by another force (effort) which in combination with other parameter gives us what we shall be discussing now. Such parameters include the true airspeed (v) and air density $(\boldsymbol{\rho})$. Some other approach will be considering, pressure (p), line integral $(\Gamma)$, wing platform area (a) and the lift coefficient $\left(C_{L}\right)$. The approaches are as follows:

*lift coefficient approach: If the lift coefficient for a wing at a specified angle of attack is known (or estimated using a method such as the thin airfoil theory), then the lift produced for specific flow is given by: $L=$ $1 / 2 V^{2} \rho a C_{L}$. This approach was tremendously utilized/applied in our work.

*kutta-Joukwski theorem: Lift can be calculated using potential flow theory by imposing a circulation. The circulation $(\boldsymbol{\Gamma})$ is the line integral of the velocity of the air in a closed loop round the boundary of an airfoil. It can be understood as the total amount of spring or vortivity (circulation) of air around the airfoil. It is said to be the section lift (L') given by: $\mathrm{L}^{\prime}=\boldsymbol{\rho} \mathrm{V} \boldsymbol{\Gamma}$.

*Pressure integration: The force on the wing can be examined in the pressure difference above and below the wings, which can be related to velocity change by Bernoulli's principle. The total lift force is the integral of vertical pressure forces over the entire wetted surface area of the wing: $L=\oint P n . k d a$

Where $\mathrm{L}$ is the lift, $\mathrm{a}$ is the wing surface area, $\mathrm{P}$ is the value of pressure, $\mathrm{n}$ is the normal unit vector pointing into the wing, and $\mathrm{k}$ is the vertical unit vector normal to the free stream direction.

\subsection{Deriving the parasitic power of the system.}

Here we shall consider what happens mathematically when an object is cruising in the air with respect to the force opposing its motion (drag). Air friction (or drag) as an example of fluid friction, unlike the standard model of surface friction, it is velocity dependent. The velocity dependence may be very complicating, hence only special cases can be treated analytically, at a very low speed for small particles, and air resistance is approximately proportional to velocity. It can be expressed in the form: 


$$
\begin{aligned}
f_{\text {drag }} & \propto V \\
& \Rightarrow f_{\text {drag }}=-b v
\end{aligned}
$$

Where the negative sign implies that it is always directly opposite the velocity, for higher velocity and bigger objects, the frictional drag is approximately proportional to the square of the velocity $(\mathrm{v})$ :

$$
f_{\text {drag }}=-1 / 2 c \rho a^{*} v^{2}
$$

Where $\boldsymbol{\rho}$ is the air density, $a^{*}$ is the cross sectional area, and c is the numerical drag coefficient.

In addition to the power been used by the wings to lift the plane, power is also required to overcome the parasitic drag (i.e. drag caused by form resistance due to shape, skin friction e.t.c.) and the induced drag. For most large high speed objects moving through fluid, the parasitic drag can be modeled as:

Where $f_{p}$ is the parasitic force (or drag)

$$
f_{p}=-1 / 2 a^{*} c \rho v^{2}
$$

But, recall that the general equation for power is given as:

So the power needed to overcome the parasitic drag is:

$$
\begin{aligned}
& p=f * \frac{s}{t} \\
& \Rightarrow p=f * v,
\end{aligned}
$$

$P_{p}=1 / 2 a^{*} c \rho v^{3}$

Where $P_{p}$ is the parasitic power, it is also the power that is counter balancing parasitic drag.

This equation shows that the power requirement of the system goes up drastically with an increase in speed. Therefore if the intention is to travel fast, then all effort should be made to streamline the object, reduce its cross-sectional area, and if possible, also travel through a low density fluid.

\subsection{Deriving the Lift power of the system.}

As the plane flies through the air, the wings are converting some of the airplane's forward thrust to throwing air down so as to lift the plane. This process of throwing air down requires energy i.e. the energy that airplane gives to the air is the kinetic type of which is: $E=1 / 2 m v^{2}$, since the airplane is continuously giving this energy to the air, it is appropriate to discuss power as the use of energy per unit time.

$$
\Rightarrow P=\frac{d E}{d t}
$$

Substituting the energy equation into the power equation gives:

Recall:

$$
P_{L}=\frac{d\left(1 / 2 m v^{2}\right)}{d t}
$$

Substitute the value of $m$ into equation (1.7)

From equation (1.8) substitute the value of $f t$.

$$
\begin{aligned}
& f=m a=\frac{m v}{t} \\
& \Rightarrow f=\frac{m v}{t} \\
& f t=m v \\
& \Rightarrow m=\frac{f t}{v}
\end{aligned}
$$

But

$$
\begin{gathered}
P_{L}=\frac{d\left(1 / 2 v^{2} \cdot \frac{f t}{v}\right)}{d t} \\
=\frac{d(1 / 2 v f t)}{d t} \\
=1 / 2\left(\frac{d v}{d t}\right) f t \\
P_{L}=1 / 2 a f t
\end{gathered}
$$

$$
\begin{array}{r}
P_{L}=1 / 2 a m v \\
a=v / t \\
\Rightarrow P_{L}=\frac{m v^{2}}{2 t}
\end{array}
$$

Where $P_{L}$ is the power needed for lift, $\mathrm{m}$ is the mass of the airplane, $\mathrm{v}$ is the velocity, and $\mathrm{t}$ is the time of flight.

\section{The general model}

In getting our general model, it is simply the addition of the power required to overcome drag (i.e. parasitic power $\left.P_{p}\right)$ and the power required for lift $\left(P_{L}\right)$, which gives us our total power needed for flight $\left(P_{T}\right)$. We know:

$P_{T}=P_{p}+P_{L}$ 
Substitute the values of $P_{p}$ and $P_{L}$ into the above equation to get our total power $\left(P_{T}\right)$.

$$
\begin{array}{r}
\Rightarrow P_{T}=1 / 2 a^{*} c \rho v^{3}+\frac{m v^{2}}{2 t} \\
P_{T}=\frac{v^{2}}{2} \cdot\left(a^{*} c \rho v+\frac{m}{t}\right) \ldots \ldots \ldots \ldots \ldots
\end{array}
$$

\subsection{Deriving the minimum velocity for flight.}

The minimum power for flight is gotten from the minimum velocity of the flight. And the minimum velocity is gotten from the partial differentiation of the total power $\left(P_{T}\right)$ in equation (1.9) with respect to velocity (v).

Dividing through by v

$$
\begin{gathered}
\frac{\partial P_{T}}{\partial v}=\frac{\partial}{\partial v} \cdot\left\{\frac{v^{2}}{2} \cdot\left(a^{*} c \rho v+\frac{m}{t}\right)\right\} \\
\text { Let } \mathrm{U}=\frac{v^{2}}{2} \quad \Rightarrow \frac{\partial u}{\partial v}=v \\
\mathrm{~S}=a^{*} c \rho v+m / t \quad \Rightarrow \frac{\partial s}{\partial v}=a^{*} c \rho
\end{gathered}
$$

Using: $\frac{\partial P_{T}}{\partial v}=U \cdot \frac{\partial s}{\partial v}+S \cdot \frac{\partial u}{\partial v}$

$$
\begin{gathered}
=\frac{v^{2}}{2} \cdot a^{*} c \rho+\left(a^{*} c \rho v+\frac{m}{t}\right) v \\
=\frac{v^{2} a^{*} c \rho}{2}+v^{2} a^{*} c \rho+\frac{m v}{t}=0 \\
=\frac{3 v^{2} a^{*} c \rho}{2}+\frac{m v}{t}=0 \\
\frac{3 v^{2} a^{*} c \rho}{2}=-\frac{m v}{t}
\end{gathered}
$$

Make $v$ the subject of the above equation

$$
\begin{aligned}
& =\frac{3 v a^{*} c \rho}{2}=-\frac{m}{t} \\
& 3 v a^{*} c \rho=-\frac{2 m}{t}
\end{aligned}
$$

$$
\begin{gathered}
v=\left(-\frac{2 m}{t}\right)\left(\frac{1}{3 a^{*} c \rho}\right) \\
=-\frac{2 m}{3 a^{*} c \rho t}=-\frac{2}{3} \cdot\left(\frac{m}{a^{*} c \rho t}\right) \\
V_{\text {min }}=\frac{2}{3} \cdot\left(\frac{m}{a^{*} c \rho t}\right) \ldots \ldots \ldots \ldots(1.1
\end{gathered}
$$

3.2 Deriving the minimum power for flight. Substitute the value of minimum velocity $\left(V_{\min }\right)$ in equation (1.10) into the total power $\left(P_{T}\right)$ in equation (1.9) will give us our minimum power for flight.

$$
\begin{gathered}
P_{T}=\frac{v^{2}}{2} \cdot\left(a^{*} c \rho v+\frac{m}{t}\right) \\
V=\frac{2}{3} \cdot\left(\frac{m}{a^{*} c \rho t}\right) \\
P_{\text {min }}=\frac{\left\{\frac{2}{3} \cdot\left(\frac{m}{a^{*} c \rho t}\right)\right\}^{2}}{2}\left\{a^{*} c \rho \cdot \frac{2}{3}\left(\frac{m}{a^{*} c \rho t}\right)+\frac{m}{t}\right\} \\
=\frac{\left\{\frac{4}{9} \cdot\left(\frac{m}{a^{*} c \rho t}\right)^{2}\right\}}{2}\left\{\frac{2 a^{*} c \rho m}{3 a^{*} c \rho t}+\frac{m}{t}\right\} \\
=\frac{2}{9} \cdot\left(\frac{m}{a^{*} c \rho t}\right)^{2}\left\{\frac{2 a^{*} c \rho m}{3 a^{*} c \rho t}+\frac{m}{t}\right\}
\end{gathered}
$$




$$
\begin{gathered}
=\frac{2 m^{2}}{\left(3 a^{*} c \rho t\right)^{2}}\left\{\frac{2 a^{*} c \rho m}{3 a^{*} c \rho t}+\frac{m}{t}\right\} \\
=\frac{4 a^{*} c \rho m^{3}}{\left(3 a^{*} c \rho t\right)^{3}}+\frac{2 m^{3}}{t\left(3 a^{*} c \rho t\right)^{2}} \\
=\frac{4 a^{*} c \rho m^{3}+\left(3 a^{*} c \rho\right) 2 m^{3}}{\left(3 a^{*} c \rho t\right)^{3}} \\
=\frac{4 a^{*} c \rho m^{3}+6 a^{*} c \rho m^{3}}{\left(3 a^{*} c \rho t\right)^{3}} \\
=\frac{10 a^{*} c \rho m^{3}}{\left(3 a^{*} c \rho t\right)^{3}} \\
=\frac{10 m^{3}\left(a^{*} c \rho\right)}{(3 t)^{3}\left(a^{*} c \rho\right)^{3}} \\
\Rightarrow P_{\min }=\frac{10}{27} \cdot \frac{m^{3}}{t^{3}\left(a^{*} c \rho\right)^{2}}
\end{gathered}
$$

Thus, the minimum power needed for a plane to move without problems.

\section{Conclusion}

In conclusion, we know that both electrical and chemical powers are required for flight, but the mechanical as well as the structural aspect of it cannot be over emphasized. That is why there are shapes that no matter the electrical power available, they cannot fly. This paper only considered mechanical aspect in discussing power needed to sustain plane in the air.

\section{Selected References}

[1] Anthony, J. (2010), Aerodynamics inverse design and shape optimization via control theory, Standard University.

[2] Gareth, H. (2010), Future aircraft fuel efficiency, Qinetic.

[3] Hurt H. Jr.(1965) aerodynamics for navel aviators, navel air system.

[4] Kelvin, P. (2009) Mathematical modeling of aircraft consumption,Eastern Washington University, Cheney.

[5] Mao, S. (2004) a computational study of the aerodynamic forces and power requirements dragonfly, China eijing University.

[6] Paul, G. (2011) flight path to sustainable aviation CSIRO, Australia.

[7] Peddly, T. (1997, Introduction to fluid dynamics, silver st. Cambridge, UK.

[8] Ray, P. (2010), aerodynamics for professional pilots ( $4^{\text {th }}$ edition).

[9] Ray, P. (2007), aerodynamics for professional pilots ( $2^{\text {nd }}$ edition). 